\title{
Mejoras en la calidad de vida de los pacientes con Miastenia Gravis sin timoma intervenidos con timectomía videotoracoscópica
}

\author{
Improvements in the quality of life of non-thymomatous Myasthenia Gravis patients \\ undergoing video thoracoscopic thymectomy
}

\author{
Nathalie C. Pinos-Vélez ${ }^{1,2}$ iD, Miguel Congregado ${ }^{3}$ \\ 1 Hospital José Carrasco Arteaga, Departamento de Cirugía de tórax, Av. 24 de mayo, Cuenca, Ecuador. \\ 2 Hospital Universitario del Río, Universidad del Azuay, Av. 24 de mayo, Cuenca, Ecuador. \\ 3 Hospital Virgen de la Macarena. Av Fedrian s/n, Sevilla, España, 41009. \\ Autora para correspondencia: nathaliepinos@ hotmail.com \\ Fecha de recepción: 6 de mayo de 2019 - Fecha de aceptación: 17 de junio de 2019
}

\begin{abstract}
RESUMEN
La Timectomía es parte del tratamiento de la Miastenia Gravis debido a que puede producir remisiones de la sintomatología. Los parámetros que determinan el éxito de la cirugía son únicamente cuantitativos: cantidad de medicación o presencia/ausencia de síntomas, sin tomar en consideración los cambios del campo psicosocial. Este estudio, de tipo cualitativo retrospectivo, determina los cambios psicosociales en los pacientes que han sido tratados con Timectomía por Miastenia Gravis no Timomatosa. Variables como sexo, edad, medicación, grupo de Osserman pre y post quirúrgico, escala de Oosterhuis, escala de Millichap y escala de la Fundación Americana de MG, fueron recolectadas de las fichas médicas de los pacientes entre 2003 al 2013. Diecisiete personas cumplieron los criterios de inclusión: Ser mayores de 18 años con diagnóstico de Miastenia Gravis no Timomatosa a los que se les realizó timectomía extendida. Estas personas fueron encuestadas telefónicamente mediante el cuestionario SF-36. Los datos recopilados se analizaron con SPSS 22.0. La calidad de vida, antes y después de la cirugía, fue comparada mediante la prueba no paramétrica de Wilcoxon. El resultado quirúrgico de los pacientes fue: $52.9 \%$ tuvo una mejoría clínica significativa de acuerdo a la escala Oosterhuis (remisión total $=5$, síntomas leves $=3$, discapacidad leve $=1$ ), de estos, el $17.6 \%$ presentó una remisión completa estable, sin medicación, y el 35.2\% presentó remisión farmacológica con dosis bajas. Los resultados del cuestionario SF-36 indican que los pacientes tratados mejoraron su nivel medio de vida $(\mathrm{p}<0.05)$ después de la timectomía.
\end{abstract}

Palabras clave: Miastenia Gravis, timectomía, calidad de vida.

\begin{abstract}
Thymectomy as part of Myasthenia Gravis treatment has proved to produce total or partial clinical remissions. But almost always, parameters to measure this outcome were quantitative, as amount of medication, presence or absence of symptoms. Very important factors of the psychosocial field as real determinants of quality of life after surgery in this kind of patients have been less investigated. Thereto, we performed a retrospective qualitative study to know the quality of life improvement after thymectomy in non-thymomatous Myasthenia Gravis patients. Seventeen patients in the period January 2003 to December 2013 met the inclusion criteria: extended thymectomy, Myasthenia Gravis confirmed, non-thymomatous, over 18 years-old. We applied the SF-36 questionnaire and the collected data were tabulated and analysed with SPSS 22.0. The Wilcoxon test for non-parametric data was used to investigate any change in the quality of life after surgery. Quantitative surgical outcome of these patients was: $52.9 \%$ had significant clinical improvement according to the Oosterhuis scale (total remission $=5$, mild symptoms $=3$, mild disability $=1$ ), of which $17.6 \%$ had complete stable remission without medication and $35.2 \%$ had pharmacological remission with low doses. All dimensions of the SF-36 questionnaire improved their median value after thymectomy, with $\mathrm{p}<0.05$. It seems that there is an improvement of quality of life in patients suffering Myasthenia Gravis after extended thymectomy.
\end{abstract}

Keywords: Myasthenia Gravis, thymectomy, quality of life.

\section{INTRODUCCIÓN}

La Miastenia Gravis (MG) es un trastorno neuromuscular, autoinmune y adquirido, caracterizado por debilidad y fatigabilidad de los músculos esqueléticos pues produce anticuerpos dirigidos contra el receptornicotínico de acetilcolina en el músculo estriado (Tapias-Vargas,
Tapias-Vargas, \& Tapias, 2009; Zenteno, Buenrostro, \& Delgadillo, 2000). La MGFA (MGFA, n.d.), cataloga a la enfermedad en cinco clases; en la primera se incluye afecciones sólo en la musculatura ocular; desde la segunda a la cuarta se incluyen afecciones musculares y oculares en nivel de afectación creciente; cada una puede subclasificarse en A y B; en A hay afección predominante de la musculatura axial y/o de extremidades, y en la 
subclase B predomina la afección en los músculos orofaríngeos, respiratorios o incluso ambos; la quinta clase hace referencia a crisis miasténica (Gómez, Álvarez, \& Puerto, 2013; Jayam Trouth, Dabi, Solieman, Kurukumbi, \& Kalyanam, 2012; Navarro-Reynoso, 2006). Osserman y Genkins proponen una clasificación de 4 niveles, ver Tabla 1 (Shields, 2005).

Desde que Oppenheim publicó el resultado de la autopsia de una paciente con miastenia en la que encontró un tumor tímico, MG ha sido relacionada con alteraciones de la glándula tímica (Jácome Roca et al., 2009; MimenzaAlvarado, Tellez-Zenteno, Garcia-Ramos, \& Estañol, 2007). Desde entonces, esta relación se ha ido fortaleciendo, de hecho, en la actualidad, la timectomía es recomendada en los pacientes jóvenes con miastenia que no responden al tratamiento médico (Camacho-Salas et al., 2002; Luis González, Pérez, \& Nodarse Fleites, 1988). La timectomía se recomienda en los primeros años posteriores al diagnóstico. El objetivo es la extirpación del timo en su totalidad, junto con la grasa peritímica, ya sea por esternotomía media, toracotomía, cervicotomía o por videotoracoscopia. En muchos casos la cirugía estabiliza la enfermedad, reduce la medicación y la incidencia de crisis miasténicas, pero este beneficio no aparece de forma inmediata tras la operación sino de forma progresiva en meses o años después (DeFilippi, Richman, \& Ferguson, 1994; Durelli et al., 1991; Loscertales et al., 2004, 1999; Maggi et al., 1989; Shields, 2005).

Varios estudios se han publicado tratando de aclarar los beneficios que esta técnica ha producido, describiendo remisiones que van desde parciales hasta totales, pero sólo han tomado en cuenta parámetros cuantitativos como la medicación, y la presencia o ausencia de sintomatología, dejando a un lado un factor muy importante como son los beneficios tangibles por el enfermo en la esfera psicosocial siendo estos los verdaderos determinantes de los cambios en la calidad de vida tras la intervención (Cakar et al., 2007; Freeman, Ascioti, Van Woerkom, Vyverberg, \& Robison, 2011; Keijzers et al., 2014; Maggi et al., 1989; Manlulu et al., 2005; Masaoka et al., 1996; Meacci et al., 2009; Park, Choi, Lee, Kim, \& Chung, 2006). El objetivo de este estudio es conocer los cambios en la calidad de vida en los pacientes afectos de MG, tras timectomía sin timoma, tratados en el Hospital Virgen de la Macarena en Sevilla, España durante el periodo 2003-2013.

\section{MATERIALES Y MÉTODOS}

La presente investigación es de tipo cualitativa, retrospectiva y descriptiva, para determinar la mejoría en la calidad de vida de los pacientes intervenidos de timectomía videotoracoscopica por Miastenia Gravis sin timoma en el Hospital Universitario Virgen de la Macarena en Sevilla España del 2003 al 2013. La aprobación de la investigación la realizó el comité de ética del Hospital Universitario Virgen Macarena de Sevilla.

Se realizó una búsqueda en los registros hospitalarios, en el estudio se incluyeron los pacientes mayores de 18 años, con diagnóstico de Miastenia Gravis a los que se le realizó una Timectomía extendida; se excluyeron pacientes con tumores tímicos (Timoma o carcinoma de Timo) y fallecidos al momento del estudio. En las fichas y otros documentos de los casos elegidos, se buscaron las variables: edad, sexo, grupo de Osserman, medicación pre y post quirúrgica, anatomía patológica definitiva, tiempo trascurrido desde el diagnóstico hasta la cirugía, escala de Oosterhuis, escala de Millichap y escala de la Fundación Americana de $\mathrm{MG}$ post quirúrgica.

Para determinar los cambios en la calidad de vida, previo consentimiento informado de los pacientes, se aplicó el cuestionario SF-36, mediante encuesta telefónica. Para las encuestas se usaron dos cuestionarios SF-36; el prequirúrgico, que incluye preguntas sobre la situación previa, y el postquirúrgico, que incluye preguntas acerca de su situación actual. Ambos, se realizaron al mismo tiempo, confiando en la memoria de los pacientes con respecto a su situación antes de la cirugía; siendo esta, la principal limitación del estudio (sesgo de memoria).

Escala de clasificación de la MG: Para determinar la afectación clínica de nuestros pacientes utilizamos la escala de Osserman y Genkins que se incluye en la Tabla 1.

Escalas Clínicas para valorar el éxito de la cirugía: Para valorar la respuesta al tratamiento quirúrgico existen diferentes escalas, entre las más importantes están la de Oosterhuis (0: remisión total, 1: signos y síntomas leves, 2: discapacidad leve, 3: discapacidad moderada, 4: discapacidad severa y 5 : requiere soporte ventilatorio), la de Millichap/Dodge (A: remisión completa sin medicación, B: buena respuesta, con dosis bajas de medicación, C: respuesta regular con dosis altas, D: sin cambios o empeoramiento y E: muerte) y la de la Fundación Americana de MG (Remisión completa estable RCE: Ausencia de síntomas por al menos un año, Remisión farmacológica RF: Ausencia de síntomas por al menos un año con el uso de algún tipo de terapia farmacológica, y Manifestaciones mínimas: el paciente no tiene síntomas de MG pero tiene debilidad en algunos músculos) (Oosterhuis, 1989; Papatestas et al., 1987).

Escala SF-36 de Calidad de Vida: El cuestionario de salud International Quality of Life Assessment (IQOLA) fue desarrollado en los noventa en Estados Unidos y fue traducido al español con el nombre de SF-36. Está compuesto por 36 preguntas (ítems) que evalúan 8 dimensiones del estado de salud: Función física, Rol físico, Dolor corporal, Salud general, Vitalidad, Función social, Rol emocional y Salud mental. Está dirigido a personas $\geq 14$ años y puede ser auto realizado o mediante entrevista (personal o telefónica). Para su evaluación los resultados son transformados en una escala que va desde 0 (el peor estado de salud) hasta 100 (el mejor estado de salud)(Alonso, Prieto, \& Antó, 1995; Garratt, Schmidt, Mackintosh, \& Fitzpatrick, 2002; Vilagut et al., 2005; Ware, 1993; Ware Jr, 2000).

Los datos colectados fueron tabulados y analizados con el programa SPSS 22.0 y, para comparar los cambios en la calidad de vida tras el tratamiento quirúrgico, se utilizó la prueba de Wilcoxon para datos no paramétricos.

\section{RESULTADOS}

El grupo de estudio estuvo conformado por un total de 17 pacientes, 13 mujeres y 4 hombres, con una edad media de $39 \pm 11.5$ años. La cirugía fue por vía toracoscópica en todos los casos, sin presentar complicaciones postquirúrgicas. La anatomía patológica fue de hiperplasia folicular en 10 pacientes $(58.8 \%)$, timolipoma en 4 
$(23.5 \%)$, atrofia en $2(11.8 \%)$ y normal en $1(5.9 \%)$. La medicación previa a la intervención fue: fármacos anticolinérgicos en 14 casos, corticoides en 13, plasmaféresis en 1 e inmunoglobulina en 1. En todos los pacientes había trascurrido más de un año desde el diagnóstico de MG hasta la cirugía. En el tiempo trascurrido en años, desde la cirugía hasta la realización del cuestionario, vemos que existe una distribución heterogénea, con un intervalo de 0 a 11 años. Encontrando $7(41.2 \%)$ pacientes con menos de 5 años y $10(58.8 \%)$ en los que han transcurrido más de 5 años de la cirugía.

Los resultados en las diferentes escalas, luego de la timectomía, se muestran en la Tabla 1, donde se ve que en la distribución por grupo de Osserman ha existido un cambio, con un valor inicial de 5 pacientes en estadio $2 \mathrm{~A}$ y 12 en 2B a un valor de 5 pacientes sin enfermedad, 6 en estadio 2A y 6 en estadio 2B. Por lo que existe un $29.4 \%$ (5 pacientes) con remisión total, una mejoría del $50 \%$ del grupo $2 \mathrm{~B}$ y del $60 \%$ del grupo $2 \mathrm{~A}$, que nos dan un total de $52.9 \%$ (9 pacientes) con mejoría clínica. En la escala de Oosterhuis se puede observar que en 5 casos existió una remisión total, 3 con síntomas leves y 1 con discapacidad leve, lo que hace un total de 9 pacientes con mejoría clínica significativa, lo que concuerda con los datos obtenidos con el grupo de Osserman. En lo que se refiere a la necesidad de medicación postquirúrgica existió una remisión completa estable (RCE) sin medicación en 3 pacientes y buena respuesta con dosis bajas en 6 (remisión farmacológica), dando un total de 9 pacientes con respuesta favorable.

Tabla 1. Escala de Osserman y Genkins para MG(Shields, 2005).

\begin{tabular}{|c|c|c|}
\hline Grupo & Afectación & Características \\
\hline 1 & Ocular (15\% a $20 \%)$ & $\begin{array}{l}\text { - } \quad \text { Limitado a músculos oculares. } \\
\text { - } \quad 40 \% \text { desarrollan enfermedad generalizada. } \\
\text { - } \quad \text { Electromiografía puede ser positiva en músculos periféricos. }\end{array}$ \\
\hline $2 \mathrm{~A}$ & $\begin{array}{l}\text { Enfermedad moderadamente } \\
\text { generalizada }(30 \%) \text {. }\end{array}$ & $\begin{array}{l}\text { - } \quad \text { Compromiso de los músculos de cabeza, miembros y tronco. } \\
\text { - } \quad \text { Músculos respiratorios conservados. } \\
\text { - } \quad \text { Buena respuesta a las drogas con anticholinesterasa. } \\
\text { - } \quad \text { Bajo mortalidad. }\end{array}$ \\
\hline $2 \mathrm{~B}$ & $\begin{array}{l}\text { Enfermedad moderadamente } \\
\text { generalizada severa }(20 \%) \text {. }\end{array}$ & $\begin{array}{l}\text { - Diplopía y ptosis significativa. } \\
\text { - Afectación de músculos bulbares: disartria, disfagia y dificultad } \\
\text { para tragar. } \\
\text { - } \quad \text { Debilidad de miembros. } \\
\text { - Intolerancia al ejercicio. }\end{array}$ \\
\hline 3 & $\begin{array}{l}\text { Enfermedad aguda } \\
\text { fulminante }(11 \%) \text {. }\end{array}$ & $\begin{array}{ll}\text { - } & \text { Inició súbito. } \\
\text { - } & \text { Los síntomas severos aparecen en } 6 \text { meses. } \\
\text { - } & \text { Afectación temprana de los músculos respiratorios. } \\
\text { - } & \text { Debilidad severa de extremidades y tronco. } \\
\text { - } & \text { Pobre respuesta a la anticolinesterasa. } \\
\text { - } & \text { Crisis frecuentes. } \\
\text { - } & \text { Alta mortalidad. } \\
\text { - } & \text { Timoma relativamente frecuente. }\end{array}$ \\
\hline$\overline{4}$ & $\begin{array}{l}\text { Enfermedad tardía severa } \\
(9 \%) .\end{array}$ & $\begin{array}{l}\text { - } \quad \text { Progresión lenta de la enfermedad después de dos años. } \\
\text { - } \quad \text { Alta incidencia de timoma. } \\
\text { - } \quad \text { Pronóstico relativamente malo. }\end{array}$ \\
\hline
\end{tabular}

Tabla 2. Dimensiones del Cuestionario SF-36.

\begin{tabular}{ll}
\hline Dimensión & Significado \\
\hline Función física & $\begin{array}{l}\text { Grado en el que la falta de salud limita las actividades físicas de la vida diaria, como el cuidado } \\
\text { personal, caminar, subir escaleras, coger o transportar cargas, y realizar esfuerzos moderados e } \\
\text { intensos. }\end{array}$ \\
Rol físico & $\begin{array}{l}\text { Grado en el que la falta de salud interfiere en el trabajo y otras actividades diarias, produciendo como } \\
\text { consecuencia un rendimiento menor del deseado, o limitando el tipo de actividades que se puede } \\
\text { realizar o la dificultad de las mismas. } \\
\text { Medida de la intensidad del dolor padecido y su efecto en el trabajo habitual y en las actividades del } \\
\text { Dolor corporal }\end{array}$ \\
halud general & $\begin{array}{l}\text { Valoración personal del estado de salud, que incluye la situación actual y las perspectivas futuras y la } \\
\text { resistencia a enfermar. }\end{array}$ \\
Vitalidad & $\begin{array}{l}\text { Sentimiento de energía y vitalidad, frente al de cansancio y desánimo. } \\
\text { Función social } \\
\text { vida social habitual. }\end{array}$ \\
Rol emocional & $\begin{array}{l}\text { Grado en el que los problemas emocionales afectan al trabajo y otras actividades diarias, considerando } \\
\text { la reducción del tiempo dedicado, disminución del rendimiento y del esmero en el trabajo. }\end{array}$ \\
Salud mental & $\begin{array}{l}\text { Valoración de la salud mental general, considerando la depresión, ansiedad, autocontrol, y bienestar } \\
\text { general. }\end{array}$ \\
\hline
\end{tabular}


Tabla 3. Resultados en la Miastenia Gravis post-timectomía.

\begin{tabular}{llcc}
\hline Escala & Grupos & Precirugía & Postcirugía \\
\hline Osserman & Sin enfermedad & 5 & 5 \\
& 2A & 12 & 6 \\
& 2B & & 6 \\
\hline Oosterhuis & 0 Remisión total & 5 \\
& 1 Signos y síntomas leves & 3 \\
& 2 Discapacidad leve & 1 \\
& 3 Discapacidad moderada & 4 \\
& 4 Discapacidad severa & 4 \\
\hline Grado de Millichap y Dodge & A Remisión completa sin medicación & 3 \\
& B Buena respuesta con medicación & & 6 \\
& C Respuesta regular con medicación & & 6 \\
& D Sin cambios o empeoramiento & 2 \\
\hline
\end{tabular}

Tabla 4. Cambios en la calidad de Vida según el Cuestionario SF-36.

\begin{tabular}{lccccc}
\hline Dimensión & Percentil & Pre-cirugía & Post-cirugía & Wilcoxon & Valor-p \\
\hline Función física & 25 & 10 & 22.5 & & \\
& 50 & 25 & 90 & $-2.728^{\mathrm{b}}$ & .006 \\
& 75 & 47.5 & 100 & & \\
\hline Rol físico & 25 & 0 & 0 & & \\
& 50 & 0 & 100 & $-2.530^{\mathrm{b}}$ & .011 \\
\hline Dolor corporal & 75 & 0 & 100 & & \\
& 25 & 22.5 & 27.5 & & \\
& 50 & 32.5 & 55 & $-2.717^{\mathrm{b}}$ & .007 \\
\hline Salud general & 75 & 37.5 & 100 & & \\
& 25 & 25 & 25 & & \\
& 50 & 30 & 65 & $-2.502^{\mathrm{b}}$ & .012 \\
\hline Vitalidad & 75 & 35 & 82.5 & & \\
& 25 & 20 & 22.5 & & \\
\hline Función social & 50 & 25 & 50 & $-2.627^{\mathrm{b}}$ & .009 \\
& 75 & 37.5 & 70 & & \\
& 25 & 12.5 & 25 & & \\
& 50 & 25 & 53 & $-2.842^{\mathrm{b}}$ & .004 \\
\hline Rol emocional & 75 & 37.5 & 87.5 & & \\
& 25 & 0 & 16.6 & & \\
Salud mental & 50 & 0 & 100 & $-2.264^{\mathrm{b}}$ & .024 \\
& 75 & 100 & 100 & & \\
& 25 & 28 & 34 & & .006 \\
\hline
\end{tabular}

Nota: Valores pre y postcirugía están expresados en medianas. Significación estadística en valores $\mathrm{p}<0.05$.

Los cambios en la calidad de vida se muestran en la Tabla 4 , donde se evidencia que hay una mejora de la mediana en todas las esferas que valora el cuestionario SF-36. En la función física vemos una elevación de la mediana que pasa del 25 al 90, en el rol físico y emocional sube del 0 al 100, en el dolor corporal pasa del 32.5 al 55 en la salud general del 30 al 65, en la vitalidad del 25 al 50, en la función social del 25 al 53 y en la salud mental del 44 al 68. Para finalizar, en la comparación entre la calidad de vida previa a la cirugía y tras la misma, se obtuvieron valores $\mathrm{p}<0.05$ en todas las dimensiones, esto es, los cambios encontrados son estadísticamente significativos.

\section{DISCUSIÓN}

La Miastenia Gravis es una patología que se diagnóstica en personas jóvenes (20-30 años), produciendo un decremento paulatino en la calidad de vida de este grupo poblacional con una disminución de las funciones en las diferentes esferas física, social, laboral y emocional. Al revisar la bibliografía nos encontramos ante la falta de estudios similares, la mayoría de las publicaciones encontradas determinan que existió una buena o mala respuesta post timectomía, basándose únicamente en la necesidad de medicación postquirúrgica y la existencia de sintomatología.

Estudios relevantes, en los que se mide la calidad de vida de los pacientes intervenidos de MG, son: el trabajo de Brush y colegas (Busch, Machens, Pichlmeier, Emskötter, \& Izbicki, 1996) y el de Bachmann (Bachmann et al., 2008), los dos realizados en Hamburgo en diferentes momentos. El primer estudio fue realizado en 1996 y su objetivo fue comparar la calidad de vida de los pacientes intervenidos de timectomia por MG que tuvieron una respuesta favorable a la cirugía, según la escala de Osserman, frente a los que no tuvieron respuesta o cambios en el postoperatorio; dicho estudio encontró una mejoría significativa en el primer grupo de pacientes. El segundo estudio, realizado en 2008, comparó la calidad de vida de pacientes, intervenidos de timectomia por MG, 
tratados mediante videotoracoscopia frente a los intervenidos por cirugía abierta. Dicho estudio, no encontró diferencias significativas entre los dos grupos. Ninguno de los trabajos diferencia los casos con timoma de los que no lo tenían, ni la vía de abordaje por lo cual se realizó la cirugía. Estos son los puntos claves de nuestro estudio, debido a que existe una clara indicación de la timectomía en pacientes con tumor tímico, con o sin presencia de MG, surgiendo la duda cuando no se evidencia dicha tumoración, al no existir en la actualidad protocolos estandarizados de manejo, por no saber con certeza hasta qué punto la cirugía podría ser beneficiosa en esta enfermedad. Además, la cirugía abierta podría determinar un decremento mayor en la calidad de vida, con respecto a la cirugía videotoracoscopica (Bachmann et al., 2008). Por lo que, a pesar de que estos estudios presentan un mayor número de pacientes y evalúan la calidad de vida al igual que nosotros, no están investigando la misma población, siendo el suyo un grupo heterogéneo términos de: indicación de la cirugía, vía de abordaje utilizada y forma de determinar la mejoría en la calidad de vida.

Como podemos ver en la Tabla 5, las cifras de remisión son muy variables, esto podría deberse a factores como la media de edad de los pacientes (más jóvenes mejor respuesta), el tiempo de diagnóstico de la enfermedad (cirugía temprana mejor respuesta), y el lapso entre la cirugía y la valoración postoperatoria (más de 5 años mejor respuesta), (Freeman et al., 2011; Maggi et al., 1989; Masaoka et al., 1996). A pesar de eso, los estudios indicados concuerdan en que existe una mejoría, tanto clínica como farmacológica, posterior a la timectomía. Dichos resultados son independientes del tipo de abordaje quirúrgico realizado, con pequeñas variaciones con respecto al índice de respuesta, que puede ser mayor o menor según la serie de casos analizada, lo cual indica que la timectomía es una estrategia terapéutica útil y que debe ser considerada como parte del arsenal terapéutico en los pacientes con MG (Maggi et al., 1989; Masaoka et al.,
1996; Park et al., 2006). Como se puede comprobar, los estudios se basan en escalas clínicas y farmacológicas para catalogar si un paciente ha tenido una mejor o peor respuesta, dejando a un lado la percepción del paciente sobre su estado de salud y su enfermedad. En cambio, nuestro estudio se basó en justamente en este último aspecto. Nuestros resultados indican una mejoría en todas las dimensiones del cuestionario SF-36. Los ítems que mayor mejoría presentaron fueron el rol físico y emocional que subieron su mediana de 0 a 100 puntos, lo que significa pasar del peor estado de salud al mejor de todos, tomando en cuenta que el rol físico es una de los más afectados en los pacientes con MG, este cambio debe ser remarcado en nuestro trabajo. Otro ítem que tuvo una elevación considerable de la mediana, de 25 a 90, fue el de la función física, que es uno de los principales factores que limitan a las personas que tienen MG. Los presentes resultados indican que si bien, según las escalas clínicas, la mejoría no fue catalogada como tal en todos los casos, para los pacientes este resultado sí cambió su calidad de vida, ayudándoles a mejorar la misma substancialmente. Además de las anteriores dimensiones, que fueron las que presentaron los cambios más llamativos, no se debe dejar atrás los cambios en la mediana del dolor corporal, que pasaron del 32.5 al 55, la salud general del 30 al 65, la vitalidad del 25 al 50, la función social del 25 al 53 y la salud mental del 44 al 68. Encontrándose significación estadística en todos los cambios encontrados en las diferentes dimensiones del cuestionario SF-36.

Para finalizar, a tenor de los resultados de nuestro estudio, sería aconsejable un estudio con un mayor tamaño muestral, para poder concluir fehacientemente que la timectomía produce un cambio importante en la calidad de vida de los pacientes con MG, por lo que nuestra serie debe ser tomada como un eslabón inicial para la realización de un proyecto de mayor envergadura que sea capaz de obtener resultados extrapolables a la población general.

Tabla 5. Estudios acerca de los resultados con escalas clínicas de la timectomía en la MG.

\begin{tabular}{|c|c|c|c|c|c|c|c|c|c|c|}
\hline \multirow[b]{2}{*}{ Variable } & \multicolumn{10}{|c|}{ Fuentes } \\
\hline & $\begin{array}{l}\text { Maggi et } \\
\text { al. (1989) }\end{array}$ & $\begin{array}{c}\text { Masaoka } \\
\text { et al. } \\
(1996)\end{array}$ & $\begin{array}{c}\text { Park } e t \\
\text { al. (2006) }\end{array}$ & $\begin{array}{c}\text { Cakar et } \\
\text { al. (2007) }\end{array}$ & $\begin{array}{l}\text { Meacci } e t \\
\text { al. (2009) }\end{array}$ & $\begin{array}{l}\text { Manlulu } \\
\text { et al. } \\
\text { (2005) }\end{array}$ & $\begin{array}{c}\text { Freeman } \\
\text { et al. } \\
\text { (2011) }\end{array}$ & $\begin{array}{l}\text { Keijzers } e t \\
\text { al. (2014) }\end{array}$ & $\begin{array}{c}\text { Diaz, } \\
\text { Black, \& } \\
\text { Dunning } \\
(2013)\end{array}$ & $\begin{array}{l}\text { Pinos } \\
\text { (este } \\
\text { trabajo) }\end{array}$ \\
\hline Lugar & Torino & Nagoya & Yonsei & Innsbruck & Roma & $\begin{array}{l}\text { Homk } \\
\text { Kong }\end{array}$ & Indiana & Maastricht & Oxford & Sevilla \\
\hline$\overline{\text { Año }}$ & 1989 & 1996 & 2006 & 2007 & 2009 & 2005 & 2011 & 2014 & 2014 & 2016 \\
\hline $\begin{array}{l}\text { Retrospectivo } \\
\text { Prospectivo }\end{array}$ & $\mathrm{X}$ & $\mathrm{X}$ & $\mathrm{X}$ & $\mathrm{X}$ & & $\mathrm{X}$ & $X$ & $\mathrm{X}$ & & $\mathrm{X}$ \\
\hline $\begin{array}{l}\text { Revisión } \\
\text { sistemática }\end{array}$ & & & & & $\mathrm{x}$ & & & & $\mathrm{x}$ & \\
\hline Muestra & 500 & 286 & 147 & 19 & 180 & 38 & 75 & 125 & 16 & 17 \\
\hline Timoma & no & no & no & sí & no & no & no & sí & sí & no \\
\hline $\begin{array}{l}\text { Remisión (\%) } \\
5 \text { años } \\
10 \text { años }\end{array}$ & & 45.8 & $\begin{array}{l}29.6 \\
45.2\end{array}$ & & 55 & & & 28.2 & $38-72$ & 17.6 \\
\hline$\frac{10 \text { años }}{\text { Mejoría (\%) }}$ & $\begin{array}{l}37.9 \\
49.4\end{array}$ & & 45.2 & 89 & & 91.6 & 87 & & & 52.9 \\
\hline $\begin{array}{l}\text { Remisión } \\
\text { farmacológic } \\
\text { a }(\%)\end{array}$ & & & & & 18.3 & & & & $6-42$ & 35.2 \\
\hline
\end{tabular}




\section{CONCLUSIÓN}

La timectomía, como tratamiento de la MG, produce una mejoría clínica con una disminución total o parcial de la medicación. Los pacientes con diagnóstico de MG, inicialmente con una baja calidad de vida, según el cuestionario SF-36, experimentaron una mejoría luego de la timectomía. Los valores de las medianas de los ítems del mencionado cuestionario se incrementaron en todas las dimensiones: función física, rol físico, rol emocional, dolor corporal, salud general, vitalidad, función social y salud mental, con una $\mathrm{p}<0.05$.

\section{REFERENCIAS}

Alonso, J., Prieto, L., \& Antó, J. M. (1995). La versión española del SF-36 Health Survey (Cuestionario de Salud SF-36): un instrumento para la medida de los resultados clínicos. Med Clin (Barc), 104(20), 771-776.

Bachmann, K., Burkhardt, D., Schreiter, I., Kaifi, J., Busch, C., Thayssen, G., ... Strate, T. (2008). Longterm outcome and quality of life after open and thoracoscopic thymectomy for myasthenia gravis: analysis of 131 patients. Surgical Endoscopy, 22(11), 2470-2477.

Busch, C., Machens, A., Pichlmeier, U., Emskötter, T., \& Izbicki, J. R. (1996). Long-term outcome and quality of life after thymectomy for myasthenia gravis. Annals of Surgery, 224(2), 225.

Cakar, F., Werner, P., Augustin, F., Schmid, T., WolfMagele, A., Sieb, M., \& Bodner, J. (2007). A comparison of outcomes after robotic open extended thymectomy for myasthenia gravis. European Journal of Cardio-Thoracic Surgery, 31(3), 501-505.

Camacho-Salas, A., Vernet, A., Colomer-Oferil, J., Pineda-Marfà, M., Campistol, J., Ribó, J. M., \& Medina, M. (2002). Timectomía en la miastenia grave juvenil. Revista de Neurología, 35(2), 119-123.

DeFilippi, V. J., Richman, D. P., \& Ferguson, M. K. (1994). Transcervical thymectomy for myasthenia gravis. The Annals of Thoracic Surgery, 57(1), 194197.

Diaz, A., Black, E., \& Dunning, J. (2013). Is thymectomy in non-thymomatous myasthenia gravis of any benefit? Interactive Cardiovascular and Thoracic Surgery, 18(3), 381-389.

Durelli, L., Maggi, G., Casadio, C., Ferri, R., Rendine, S., \& Bergamini, L. (1991). Actuarial analysis of the occurrence of remissions following thymectomy for myasthenia gravis in 400 patients. Journal of Neurology, Neurosurgery \& Psychiatry, 54(5), 406411.

Freeman, R. K., Ascioti, A. J., Van Woerkom, J. M., Vyverberg, A., \& Robison, R. J. (2011). Long-term follow-up after robotic thymectomy for nonthymomatous myasthenia gravis. The Annals of Thoracic Surgery, 92(3), 1018-1023.

Garratt, A., Schmidt, L., Mackintosh, A., \& Fitzpatrick, R. (2002). Quality of life measurement: bibliographic study of patient assessed health outcome measures. Bmj, 324(7351), 1417.
Gómez, S., Álvarez, Y., \& Puerto, J. A. (2013). Miastenia Gravis: una visión actual de la enfermedad. Medicas UIS, 26(3), 13-22.

Jácome Roca, A., Siachoque, H., Otero Ruiz, E., GómezGutiérrez, A., Quintana López, G., Pons-Estel, B., ... Iglesias-Gamarra, A. (2009). The history of organspecific diseases. The endocrine connection. Revista Colombiana de Reumatología, 16(3), 276-299.

Jayam Trouth, A., Dabi, A., Solieman, N., Kurukumbi, M., \& Kalyanam, J. (2012). Myasthenia gravis: a review. Autoimmune Diseases, 2012, 874680. https://doi.org/10.1155/2012/874680

Keijzers, M., de Baets, M., Hochstenbag, M., AbdulHamid, M., zur Hausen, A., van der Linden, M., ... Dingemans, A.-M. C. (2014). Robotic thymectomy in patients with myasthenia gravis: neurological and surgical outcomes. European Journal of CardioThoracic Surgery, 48(1), 40-45.

Loscertales, J., Jarne, J. A., Congregado, M., Tristán, A. A., Merchán, R. J., Arjona, J. G., \& Linares, C. A. (2004). Timectomía videotoracoscópica para el tratamiento de la miastenia gravis. Archivos de Bronconeumología, 40(9), 409-413.

Loscertales, J., Merchán, R. J., Linares, C. A., Díaz, F. G., Arjona, J. G., Loscertales, M. C., ... Ayuso, G. I. (1999). Tratamiento de la miastenia grave mediante timectomía videotoracoscópica. Técnica y resultados iniciales. Archivos de Bronconeumología, 35(1), 9-14.

Luis González, S., Pérez, L. R., \& Nodarse Fleites, A. (1988). Valoración de la timectomia en el tratamiento de la miastenia grave. Revista Cubana de Medicina, 27(12), 64-72.

Maggi, G., Casadio, C., Cavallo, A., Cianci, R., Molinatti, M., \& Ruffini, E. (1989). Thymectomy in myasthenia gravis. European Journal of CardioThoracic Surgery, 3(6), 504-511.

Manlulu, A., Lee, T. W., Wan, I., Law, C. Y., Chang, C., Garzon, J. C., \& Yim, A. (2005). Video-assisted thoracic surgery thymectomy for nonthymomatous myasthenia gravis. Chest, 128(5), 3454-3460.

Masaoka, A., Yamakawa, Y., Niwa, H., Fukai, I., Kondo, S., Kobayashi, M., ... Monden, Y. (1996). Extended Thymectomy for Myasthenia Gravis Patients: A 20Year Review. The Annals of Thoracic Surgery, 62(3), 853-859. https://doi.org/10.1016/S00034975(96)00376-1

Meacci, E., Cesario, A., Margaritora, S., Porziella, V., Tessitore, A., Cusumano, G., ... Granone, P. (2009). Thymectomy in myasthenia gravis via original videoassisted infra-mammary cosmetic incision and median sternotomy: long-term results in 180 patients. European Journal of Cardio-Thoracic Surgery, 35(6), 1063-1069.

MGFA. (n.d.). The Myasthenia Gravis Foundation of America (MGFA). Retrieved May 3, 2019, from http://myasthenia.org/

Mimenza-Alvarado, A., Tellez-Zenteno, J., GarciaRamos, G., \& Estañol, B. (2007). The history of myasthenia gravis. Men and ideas. Neurologia, 22, 1-8.

Navarro-Reynoso, F. (2006). Resultados de la timectomía máxima en miastenia gravis. Experiencia de 20 años en el Servicio de Neumología y Cirugía de Tórax en el Hospital General de México. Influencia 
de la timectomía en dosis de piridostigmina y tres variables espirométricas en mia. 19, 6.

Oosterhuis, H. J. (1989). The natural course of myasthenia gravis: a long term follow up study. Journal of Neurology, Neurosurgery \& Psychiatry, 52(10), 1121-1127.

Papatestas, A. E., Genkins, G., Kornfeld, P., Eisenkraft, J. B., Fagerstrom, R. P., Pozner, J., \& Aufses Jr, A. H. (1987). Effects of thymectomy in myasthenia gravis. Annals of Surgery, 206(1), 79.

Park, I. K., Choi, S. S., Lee, J. G., Kim, D. J., \& Chung, K. Y. (2006). Complete stable remission after extended transsternal thymectomy in myasthenia gravis. European Journal of Cardio-Thoracic Surgery, 30(3), 525-528.

Shields, T. W. (2005). General thoracic surgery. Lippincott Williams \& Wilkins.
Tapias-Vargas, L., Tapias-Vargas, L. F., \& Tapias, L. (2009). Miastenia gravis y el timo: pasado, presente y futuro. Revista Colombiana de Cirugía, (24), 269282.

Vilagut, G., Ferrer, M., Rajmil, L., Rebollo, P., Permanyer-Miralda, G., Quintana, J. M., ... Alonso, J. (2005). El cuestionario de salud SF-36 español: una década de experiencia y nuevos desarrollos. Gaceta Sanitaria, 19(2), 135-150.

Ware, J. E. (1993). Scoring the SF-36. SF-36. Health Survey: Manual and Interpretation Guide.

Ware Jr, J. E. (2000). SF-36 health survey update. Spine, 25(24), 3130-3139.

Zenteno, J. F. T., Buenrostro, L. E. M., \& Delgadillo, A. T. (2000). Patogénesis de la miastenia gravis. $L a$ Revista de Investigación Clínica, 52(1), 80-85. 